\title{
Multihop Cellular: A Novel Architecture for Wireless Data Communications
}

\author{
Yu-Ching Hsu and Ying-Dar Lin
}

\begin{abstract}
This work presents a novel architecture, Multihop Cellular Network (MCN), for wireless communications. MCN preserves the benefit of conventional single-hop cellular networks (SCN) where the service infrastructure is constructed by fixed bases or access points (APs), and it also incorporates the flexibility of ad-hoc networks where wireless transmission through mobile stations in multiple hops is allowed. MCN can reduce the required number of bases or APs and improve the throughput performance, while limiting path vulnerability encountered in ad-hoc networks. In addition, MCN and SCN are analyzed, in terms of mean hop count, hopby-hop throughput, end-to-end throughput, and mean number of channels (i.e., simultaneous transmissions) under different traffic localities and transmission ranges. Numerical results demonstrate that throughput of MCN exceeds that of $\mathrm{SCN}$ and the former also increases as the transmission range decreases. The above results can be accounted for by the different orders, linear and square, at which mean hop count and mean number of channels increase, respectively.
\end{abstract}

Index Terms: Multihop, cellular, ad-hoc networks, packet radio, transmission range.

\section{INTRODUCTION}

Wireless data communications has rapidly evolved in the recent decade. The services can be categorized as either (1) lowspeed, wide-area systems, e.g., CDPD (Cellular Digital Packet Data) [1] and GPRS (General Packet Radio Service) [2], or (2) high-speed, local-area systems, e.g., HIPERLAN (Hi Performance Radio Local Area Network) [3] and IEEE 802.11 [4]. The goal of HIPERLAN is to ensure wireless peer-to-peer communications at the rate comparable to Ethernet without the need for central control.

However, most services and systems mentioned above, except HIPERLAN, are based on the single-hop cellular architecture, where many fixed bases or APs are constructed to encompass the service area. In a densely populated metropolitan area, to support more connections, the area that a single base or AP covers is shrunk and the number of bases/APs increases. This phenomenon unfortunately leads to (1) a high cost for building a large number of bases/APs, (2) total throughput limited by the number of cells in an area, and (3) higher transmission power of the base/AP and mobile stations. Notably, (1) and (2) trade off each other.

Another kind of network, commonly referred to as packet radio or ad-hoc networks [5], [6], is available in which no infras-

Manuscript received October 1, 2001.

The authors are with Department of Computer and Information Science, National Chiao Tung University, Hsinchu, Taiwan, e-mail: ychsu@ cis.nctu.edu.tw, ydlin@cis.nctu.edu.tw. tructure or wireline backbone is required. In these networks, packets are routed in multihop. Second generation packet radio networks, such as WAMIS (Wireless Adaptive Mobile Information System [7]), have began to address the limited bandwidth and QoS(Quality of Service) issue. The advantages of these networks are their low cost and fast deployment. The negative side of these networks is that paths are much more vulnerable due to station mobility. However, this vulnerability can be significantly reduced if the number of wireless hops can be reduced and the station mobility is low.

In this work, we present a novel hybrid architecture, MCN (Multihop Cellular Network), derived from ad-hoc networks and the traditional SCNs (Single-hop Cellular Networks). Notably, MCN is designed for densely connected networks. The implemented prototype, where mobile stations run a bridging protocol, shows that MCN is a feasible architecture for wireless LANs [8]. In MCN, the ability of relaying packets of mobile stations is not allowed in other variant systems of SCN, such as Ricochet network [9] and mobile base network [10]. There is a similar architecture in ODMA (Opportunity Driven Multiple Access) [11], which sits upon a radio sub-system that supports relaying. However, it does not formally demonstrate that it has potential for increasing the overall system capacity, as we will investigate in this paper. MCN has several merits: (1) the number of bases/APs can be reduced to decrease the cost or the transmission power of both mobile stations and bases/APs can be lowered to be good for health, (2) connections are still allowed without bases/APs, (3) multiple packets can be simultaneously transmitted within a cell, and (4) paths are less vulnerable than the ones in ad-hoc networks because the bases/APs can help reduce the wireless hop count. The base/AP in the center of an $\mathrm{MCN}$ is referred to herein as the "base."

Fig. 1 shows the two basic methods to construct $\mathrm{MCN}$ from $\mathrm{SCN}$. One is MCN-b, where the number of bases is reduced such that the distance between two neighboring bases becomes $k_{b}$ times of that in $\mathrm{SCN}$. The example in Fig. 1 shows that to construct the MCN-b with $k_{b}=2$, only the four bases, A, B, C, and D in the SCN are necessary. The other one is MCN-p, where the transmission range of both bases and mobile stations is reduced to $1 / k_{p}$ of that in SCN. In both cases, a base is not always reachable from a mobile station in a single hop. Hence, multihop routing is necessary. Nevertheless, MCN-b can be viewed as a special case of MCN-p. For the example in Fig. 1, the radius of a cell and the distance between bases in MCN-b are both twice as long as those in MCN-p, i.e., $k_{p}=k_{b}=2$. This work focuses on MCN-p while describing and analyzing the proposed architecture. MCN-p is referred to herein as MCN.

This work focuses mainly on system throughput of SCN and $\mathrm{MCN}$. The negative side of MCN is that the throughput might 


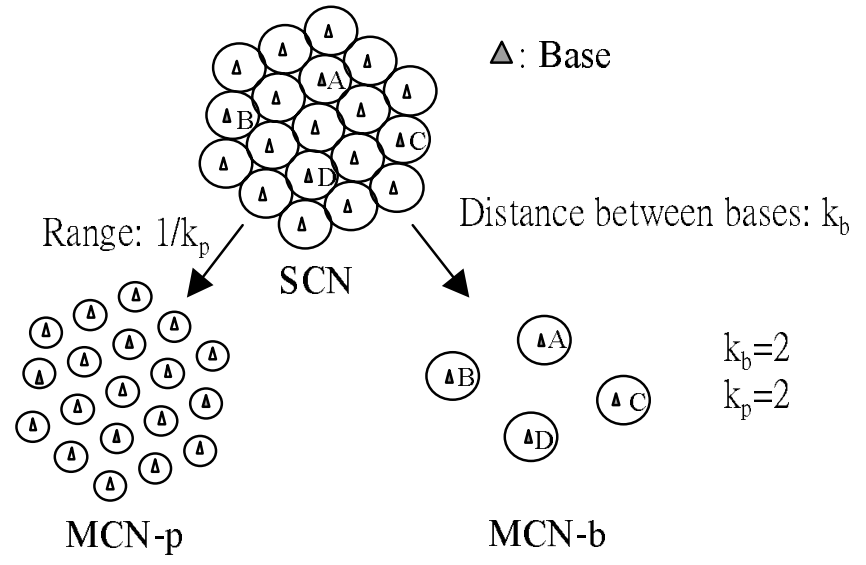

Fig. 1. Examples of an SCN and two variants of MCN, MCN-p and MCN-b.

be lowered because packets may have to be forwarded multiple times to arrive at destinations. However, the positive side is that the throughput should be improved because multiple packets can be transmitted simultaneously in a cell. We need to see whether the negative side or the positive side dominates. Thus, we analyze and compare the throughput in SCN and MCN. Herein, hop count is defined as the number of times a packet needs to be forwarded to reach its destination. The number of channels is defined as the number of packets that can be transmitted simultaneously in a cell. The results have shown that the throughput in $\mathrm{MCN}$ increases linearly as transmission range decreases. This is because mean hop count increases in linear order and the number of channels increases in square order. That is the positive side dominates.

The rest of the paper is organized as follows. Section II describes the architectures of SCN and MCN. Section III presents the system throughput of SCN and MCN. Section IV summarize the numerical results, indicating that the throughput of $\mathrm{MCN}$ is better than that of SCN. Hop count and the number of channels are also studied to explain the results. Finally, conclusion is given in section $\mathrm{V}$.

\section{ARCHITECTURE}

Single-hop Cellular Networks (SCNs) are cellular networks where bases can be reached by mobile stations in a single hop, in contrast to Multihop Cellular Networks (MCNs) where bases can not always be reached by mobile stations in a single hop. Before describing these two architectures, we first define a cell as the area taken care of by a base, which is within a radius of fixed distance, say $R$. Notably, $R$ in MCN equals half the distance between two neighboring bases. A mobile station is associated with the base within the same cell. A sub-cell is defined as the area reachable in a single wireless hop either by a base or by a mobile station. Notably, in SCN, the area of a sub-cell is the same as the area of a cell.

\section{A. Single-hop Cellular Network (SCN)}

In $\mathrm{SCN}$, the base and mobile stations in the same cell are always mutually reachable in a single hop. When having packets

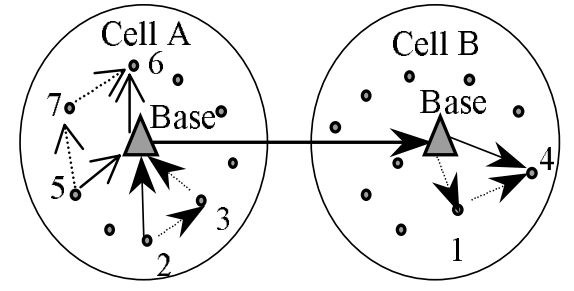

Fig. 2. Different routing paths of MCN and SCN for intra-cell and intercell traffics.

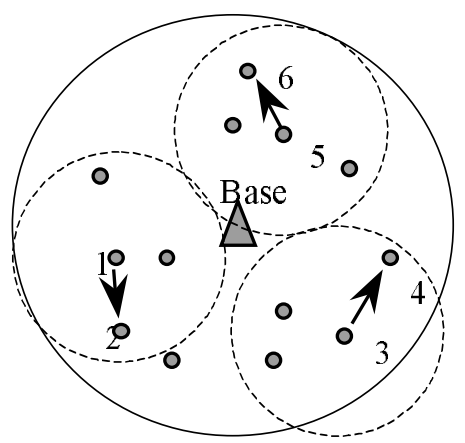

Fig. 3. Three simultaneous transmissions within a cell in MCN.

to send, mobile stations always send them to the associated base. If the destination and the source are in the same cell, such as stations 5 and 6 in Fig. 2, the base directly forwards packets to the destination. If the destination is in a different cell, the base forwards the packets to the base of the cell where the destination resides. The base of the latter cell then forwards packets to the destination in a single hop. Thus, the routing path resembles the solid lines in Fig. 2.

\section{B. Multihop Cellular Network (MCN)}

The architecture of MCN resembles that of SCN except that the transmission range of bases and mobile stations is reduced. Hence, the base and mobile stations are not always mutually reachable in a single hop. Thus, the area reachable by a base or by a mobile station, i.e., the area of a sub-cell, is smaller than the area of a cell. Similar to ad-hoc networks, a key feature of MCN is that mobile stations can directly communicate with each other if they are mutually reachable, which is not allowed in SCN. This feature leads to multihop routing.

If the source and the destination are in the same cell, other mobile stations are used to relay packets to the destination, which achieves multihop routing within a cell. If not in the same cell, packets are sent to the base first, probably in multiple hops, and then be forwarded to the base with which the destination is associated. Packets are then forwarded to the destination, probably in multiple hops again, within the latter cell.

The dotted lines in Fig. 2 illustrate the above two cases. This figure reveals the different routing paths in SCN and MCN by solid and dotted lines, respectively. The main advantage of MCN is the increased system throughput, as analyzed in the next section. For the example in Fig. 3, stations 1, 3, and 5 within the same cell can transmit packets simultaneously without interfering with each other; meanwhile, only one packet can be transmitted in the corresponding SCN. 


\section{MAC protocol}

The RTS/CTS (Request To Send/ Clear To Send) access method of DCF (Distributed Coordination Function) of IEEE 802.11 MAC (Medium Access Control) protocol is applied to MCN to be compared with SCN, because the architecture of SCN is actually the same as those of existing Wireless data LANs, in which RTS/CTS is the underlying MAC protocol. Hence, the need for synchronization is eliminated.

We also assume that neighboring cells use different channels to avoid conflict. Furthermore, to hold the spirit of RTS/CTS, i.e., to avoid the hidden terminal problem [12], the transmission range of the base and mobile stations is the same, which is reduced to $1 / k_{p}$ of that adopted in SCN. If only the transmission range of mobile station is reduced, i.e., the transmission range of the base is larger than mobile stations, serious collisions will be caused. For the example in Fig. 3, because the base is hidden from all the transmitters, i.e., 1, 3, and 5, and all the receivers, i.e., 2, 4, and 6, any packet fired by the base will cause collisions.

We assume that the up-link and down-link of MSs and the base share the same channel, i.e., frequency band. If several channels are used, a channel assignment protocol will be necessary and complicates the system. For the example in Fig. 3, if station 1 transmits in $\operatorname{ch} 1$ and listens to $\operatorname{ch} 2$, station 2 would have to listen to $c h 1$ and transmit in another channel. Thus, another control channel would be indispensable to process the channel assignment when a mobile station transmits and receives packets in separate channels, making our analysis intractable. In fact, when an MCN is derived from IEEE 802.11 WLAN, the up-link and down-link essentially share the same channel.

\section{MODELING AND ANALYSIS}

\section{A. Underlying Assumptions and Definitions}

For simplicity, the analysis process is based on the following underlying assumptions. (1) The well-known RTS/CTS access method is the underlying MAC protocol. (2) Neighboring cells use different channels to avoid interference. (3) The transmission range of the base and mobile station is the same. (4) Uplink and down-link share the same channel. (5) Station mobility is neglected because the simulation results in [13] show the small impact of mobility on system throughput. (6) The mobile stations are uniformly distributed within the cell. (7) In MCN, to simplify the analysis, the base does not forward intra-cell traffic. (8) In MCN, we assume that station $i$ can always find the next hop, at a distance of $R / k_{p}$, in the straight-line direction toward destination. For convenient sake, this assumption is noted as Route. Notably, Route is removed in our simulation to exam its impact.

The related parameters used in analysis process are defined as following. Once a channel is sensed idle and a time interval DIFS (DCF Inter-Frame Space) has elapsed, the time until an intra-cell data packet is generated at station $i$ is assumed to be exponentially distributed with rate $\lambda_{i j}$. Also, $\lambda_{i o}$ is denoted as the rate at which inter-cell data packets are generated at station $i$. Similarly, $\lambda_{o i}$ is the rate at which inter-cell data packets are

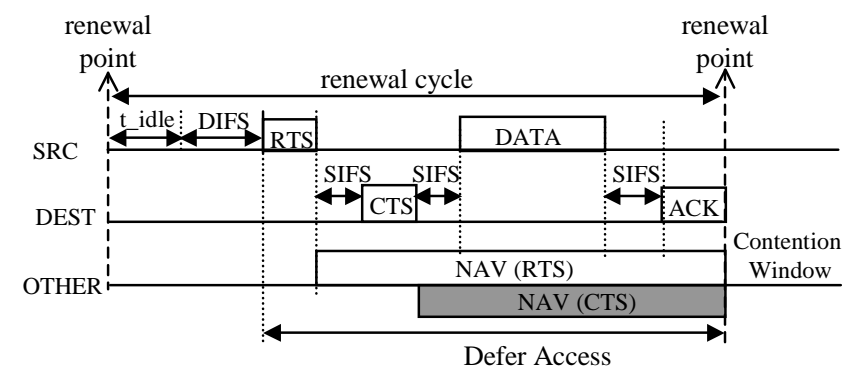

Fig. 4. The length of a renewal cycle and the operation of RTS/CTS access method.

generated outside the cell and destined for station $i$ in the cell. The number of stations in the cell is denoted as $N$ and the radius of the cell is denoted as $R$. In addition, $r, l_{R T S}, l_{C T S}$, $l_{P K T}, l_{A C K}, l_{S I F S}$, and $l_{D I F S}$ are used to denote the maximum propagation delay in one hop, the transmission time of an RTS packet, a CTS packet, a data packet and an acknowledgment packet, as well as the time interval of SIFS (Short InterFrame Space) and DIFS, respectively.

We use the technique of renewal process to model the system. Herein, the system throughput is defined using the same definition as [14], i.e., the number of successful transmissions between successive "renewal points" divided by the length of the time interval between the renewal points, which is referred to as a renewal interval. Furthermore, the "renewal point" is defined as the time point when all stations in a sub-cell simultaneously sense the channel being idle. Fig. 4 shows the operation of RTS/CTS access method and the length of a renewal cycle. The $N A V$ in the figure stands for Net Allocation Vector. Notably, the propagation delay, $r$, is not illustrated in the figure. Thus, the expected length of a renewal interval is defined as

$$
\begin{aligned}
& r n_{-} c y c l e= \\
& \quad l_{D I F S}+t_{-} i d l e+l_{R T S}+r+l_{S I F S}+l_{C T S}+r \\
& +P s *\left(l_{S I F S}+l_{P K T}+r+l_{S I F S}+l_{A C K}+r\right),
\end{aligned}
$$

where $P s$ denotes the probability of a successful exchange of an RTS and an CTS packets, and $t \_i d l e$ represents the expected time until the initiation of the first transmission since a renewal point. Notably, both $t \_i d l e$ and $P s$ are to be derived.

Our goal is to derive the hop-by-hop throughput and end-toend throughput in a cell for both SCN and MCN. The hop-byhop throughput is defined as the number of successful packet transmissions per second in a cell. The end-to-end throughput is defined as the number of successful packet receptions per second in a cell by the end destinations. We use table 1 to summarize the parameters mentioned above.

\section{B. Single-hop Cellular Network}

\section{System throughput analysis:}

To model the SCN throughput by renewal process, four major steps are followed:

1. Derive $t$ _idle to obtain the renewal interval;

2. Derive the probabilities of a successful transmission at station $i$ and at the base;

3. Derive the throughput of station $i$ and the base; and 
Table 1. Summary of parameters.

\begin{tabular}{|c|l|}
\hline$k_{p}$ & $\begin{array}{l}\text { the transmission range in MCN-p is } 1 / k_{p} \text { of the range in } \\
\text { SCN }\end{array}$ \\
\hline$k_{b}$ & $\begin{array}{l}\text { the distance between two neighboring bases in MCN-b is } \\
k_{b} \text { times of the distance in SCN }\end{array}$ \\
\hline$\lambda_{i j}$ & $\begin{array}{l}\text { packet generation rate at station } i \text { to station } j \text { in the same } \\
\text { cell }\end{array}$ \\
\hline$\lambda_{i o}$ & $\begin{array}{l}\text { packet generation rate at station } i \text { to stations outside the } \\
\text { cell }\end{array}$ \\
\hline$\lambda_{o i}$ & $\begin{array}{l}\text { packet generation rate at stations outside the cell and des- } \\
\text { tined for station } i \text { in the cell }\end{array}$ \\
\hline$N$ & the total number of stations in a cell \\
\hline$R$ & the radius of a cell \\
\hline$r$ & the maximum propagation delay in a hop \\
\hline$l_{R T S}$ & the transmission time of a RTS packet \\
\hline$l_{C T S}$ & the transmission time of a CTS packet \\
\hline$l_{P K T}$ & the transmission time of a data packet \\
\hline$l_{A C K}$ & the transmission time of an acknowledgment packet \\
\hline$l_{S I F S}$ & the time interval of SIFS \\
\hline$l_{D I F S}$ & the time interval of DIFS \\
\hline$r n_{-} c y c l e$ & the expected length of a renewal interval \\
\hline$t_{-} i d l e$ & $\begin{array}{l}\text { the expected time until the initiation of the first transmis- } \\
\text { sion since a renewal point }\end{array}$ \\
\hline$G_{s}$ & the total packet arrival rate at mobile stations within a cell \\
\hline$G_{b s}$ & the packet arrival rate at the base \\
\hline$P_{s}$ & $\begin{array}{l}\text { the probability of successful exchange of RTS and CTS } \\
\text { packets }\end{array}$ \\
\hline
\end{tabular}

4. Derive the hop-by-hop throughput and end-to-end throughput of SCN.

To obtain $r n \_c y c l e$, the packet arrival rate at station $i$ is computed as

$$
\lambda_{i}=\sum_{j \neq i, j=1}^{N} \lambda_{i j}+\lambda_{i o} .
$$

Now we denote the total packet arrival rate at all mobile stations within a cell by $G_{s}$ which equals $\sum_{i=1}^{N} \lambda_{i}$. Since each packet transmitted from station $i$ to station $j$ is forwarded by the base, the traffic generation rate at the base is given by $G_{b s}=\sum_{i=1}^{N} \lambda_{o i}+\sum_{i=1}^{N} \sum_{j \neq i, j=1}^{N} \lambda_{i j}$. Thus, $t_{-} i d l e$ is derived as $1 /\left(G_{s}+G_{b s}\right)$, since the packet arrival process is Poisson and a sub-cell equals a cell in SCN. The renewal interval is thus given by

$$
\begin{aligned}
& r n_{-} \text {cycle }= \\
& \quad l_{D I F S}+\frac{1}{G_{s}+G_{b s}}+l_{R T S}+r+l_{S I F S}+l_{C T S}+r \\
& +P s *\left(l_{S I F S}+l_{P K T}+r+l_{S I F S}+l_{A C K}+r\right) .
\end{aligned}
$$

To analyze the probability of a successful transmission from station $i$ to the base, we define capture area and hidden area of station $i$. As shown in Fig. 5, the capture area of station $i$, i.e., $\mathrm{A} 1$, is the area within the cell reachable by station $i$. The hidden area of station $i$ is the capture area of the receiver, i.e., the base station, but is hidden from station $i$, i.e., A2. For convenience, $A 1 / \pi R^{2}$ and $A 2 / \pi R^{2}$ are denoted as $n \_h$ (i.e., mean percentage of capture area in a cell) and $h$ (i.e., mean percentage of hidden area in a cell), respectively, where $\pi R^{2}$ represents the area of the cell. Since

$P($ the distance between the base and station $i=a)=\frac{2 a}{R^{2}}$,

$A 1$ equals $\int_{0}^{R} \frac{2 a}{R^{2}} \cdot\left[2 R^{2} \theta-R a \sin \theta\right] d a$, where $\theta=\arccos \frac{a}{2 R}$

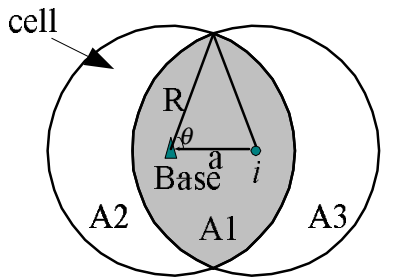

A1: capture area of station $i$ A2: hidden area of staton $i$ for the base

Fig. 5. Capture area and hidden area of station $i$ in SCN.

and $\sin \theta=\sqrt{R^{2}-\left(a^{2} / 4\right)} / R$. Thus A2 is given by $\pi R^{2}-A 1$. Notably, A3 belongs to another cell using a different channel.

Under the condition that station $i$ is the first one who fires an RTS packet, at time $t$, within its sub-cell, following are the conditions for a successful transmission from station $i$ to the base. Notably, as long as the exchange of RTS and CTS succeeds, the transmission of the following data and acknowledgement are guaranteed to be success. This is also how RTS/CTS works. Thus, only the time interval between $t$ and the time point when the corresponding CTS arrives at station $i$ needs to be considered.

(a) Station $i$ has a packet to send at time $t$;

(b) No stations in area A1 send packets during $[t, t+r]$;

(c) The base does not send packets during $[t, t+r]$; and

(d) No stations in area A2 send packets during $[t, t+r+$ $\left.l_{R T S}+l_{S I F S}+r\right]$.

Conditions (b) and (c) imply that the RTS packet will arrive in the base successfully, since no transmission occurs during $[t, t+$ $r]$ in the capture area of station $i$. After time $t+r$, all stations in A1 area will reserve the bandwidth for station $i$, where $r$ is the propagation time. Condition (d) ensures that no collision occurs due to the hidden terminal problem, since the base is supposed to reply the corresponding CTS packet at time $t+r+l_{R T S}+l_{S I F S}$. Similarly, after time $t+r+l_{R T S}+l_{S I F S}+r$, all stations in A2 area will reserve the bandwidth for the base. Hence, a pair of RTS and CTS packets is successfully exchanged. Note that the transmission in A3 area will not cause interference because the stations in A3 area belong to another cell using a different channel.

It is relatively simpler to obtain the probability of successful transmission at the base because no station in the cell is hidden from the base. Under the condition that the base is the first one who fires an RTS packet within its sub-cell. For the same reason discussed above, only the following two conditions need to be satisfied to complete the exchange of RTS and CTS:

(e) The base has a packet to send at time $t$; and

(f) No stations in the cell send packets during $[t, t+r]$.

According to above discussion, the probabilities of a successful transmission at station $i$ and at the base, $P s_{i}$ and $P s_{b s}$, is written as $\left[1-\left(1-e^{-\left(G_{b s}+G_{s} \cdot n_{-} h\right) \cdot r}\right)\right] \cdot[1-(1-$ $\left.\left.e^{-\left(G_{s} \cdot h\right) \cdot\left(2 r+l_{R T S}+l_{S I F S}\right)}\right)\right]$, i.e., $P[$ both conditions $(b)$ and $(c)$ hold $] \cdot P[$ condition $(d)$ holds $]$, and $\left[1-\left(1-e^{-G_{s} \cdot r}\right)\right]$, respectively. Thus, we obtain

$$
\begin{aligned}
P s_{i}= & \exp \left\{-\left[r \cdot\left(G_{b s}+G_{s} \cdot n_{-} h\right)\right.\right. \\
& \left.\left.+\left(2 r+l_{R T S}+l_{S I F S}\right) \cdot G_{s} \cdot h\right]\right\}
\end{aligned}
$$


and

$$
P s_{b s}=\exp \left\{-r \cdot G_{s}\right\} .
$$

With the success probabilities, we can derive the throughput pumped by station $i$ and the base as

$$
S_{i}=\frac{1 \cdot P s_{i}}{r n_{-} c y c l e} \cdot \frac{\lambda_{i}}{G_{b s}+G_{s} \cdot n \_h}
$$

and

$$
S_{b s}=\frac{1 \cdot P s_{b s}}{r n_{-} c y c l e} \cdot \frac{G_{b s}}{G_{b s}+G_{s}},
$$

respectively, where $\lambda_{i} /\left(G_{b s}+G_{s} \cdot n_{-} h\right)$ and $G_{b s} /\left(G_{b s}+G_{s}\right)$ are the probabilities for station $i$ and the base to fire the first RTS after a renewal point, respectively.

Eventually, the total hop-by-hop throughput in a cell of SCN is obtained as

$$
S_{h}=\sum_{i=1}^{N} S_{i}+S_{b s} .
$$

It is just the total throughput pumped by all mobile stations and the base in the cell. However, the end-to-end throughput of SCN equals the hop-by-hop throughput of the base, i.e.,

$$
S_{e}=S_{b s}
$$

because when the base sends a packet, the receiver is simply the end destination.

\section{Multihop Cellular Network}

Because in MCN the offered traffic within a cell is contributed by initially transmitted packets and forwarded packets, the aggregated traffic depends on mean hop count. Thus, we first need to derive mean hop count from station $i$ to either station $j$ or the base, in the same cell. To simplify the analysis for mean hop count, we make an assumption, Route, that station $i$ can always find the next hop, at a distance of $R / k_{p}$, in the straight-line direction towards the destination. In [15], the analyzed forward progress is based on a different network model and hence can not be adopted in MCN.

Hop count analysis:

Assume that station $j$ is the destination of the intra-cell packets fired by station $i$. The distribution of hop count, $h c$, from station $i$ to $j$ varies with the location of station $i$. Thus, to compute mean hop count, the distribution of the location of station $i$ should also be analyzed. The basic idea is to compute the distribution of the hop count from station $i$ to $j$ when station $i$ is located in a specific area. Then compute the mean of all distributions of hop count with different location of station $i$.

The possible location of station $i$ relative to the base can be divided into circular layers. The probability that station $i$ is in the $n$th layer of the cell, i.e., the hop count from station $i$ to the base is $n$, as the gray area shown in Fig. 6, is $\pi\left[\left(\frac{n R}{k_{p}}\right)^{2}-\right.$ $\left.\left(\frac{(n-1) R}{k_{p}}\right)^{2}\right] / \pi R^{2}$, which equals $(2 n-1) / k_{p}^{2}$, where $1 \leq n \leq k_{p}$.

Under the condition that station $i$ is positioned in the $n$th layer of the cell, the possible location of station $j$ relative to $i$ can be divided into circular layers again. As shown in Fig. 7, depending on the location of station $j$, station $i$ has four cases to consider.

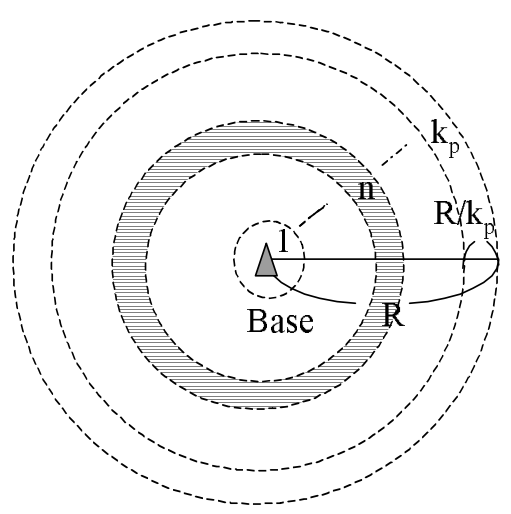

Fig. 6. The distribution of station $i$ 's position.

Legends: $0 \quad$ station $i$

$\Delta$ base

shaded area: possiable location of station $j$

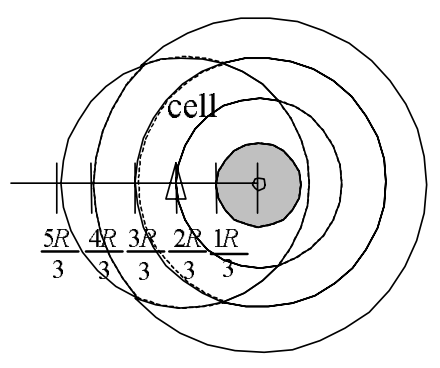

case I

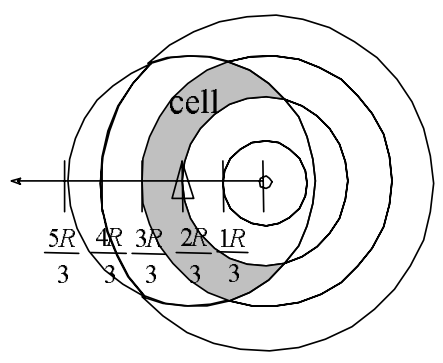

case III

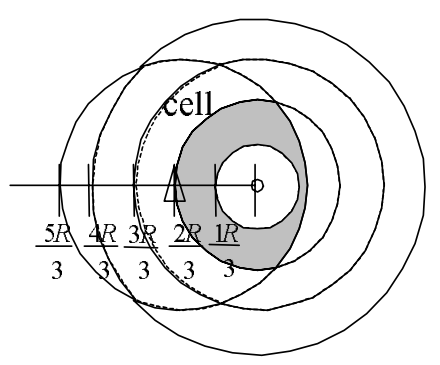

case II
Fig. 7. Four cases to compute $P($ hop count $=h c)$, for $k_{p}=3$ and $n=2$.

We compute the gray areas respectively. Then we can obtain the probability for $j$ to fall into the $h c$ th layer, i.e., $P$ (hop count $=$ $h c)$. The four cases are computed as below:

- case I: $P($ hop count $=h c)=\pi\left[\left(h c \cdot R / k_{p}\right)^{2}-((h c-\right.$ $\left.\left.1) \cdot R / k_{p}\right)^{2}\right] / \pi R^{2}$, which equals $(2 h c-1) / k_{p}^{2}$, when $1 \leq$ $h c \leq k_{p}-n$;

- case II: $P($ hop count $=h c)=\left(A\left(h c, n, k_{p}, R\right)-\pi\right.$. $\left.\left((h c-1) \cdot R / k_{p}\right)^{2}\right) / \pi R^{2}$, when $h c=k_{p}-n+1$

- case III: $P(h o p$ count $=h c)=\left(A\left(h c, n, k_{p}, R\right)-\right.$ $\left.A\left(h c-1, n, k_{p}, R\right)\right) / \pi R^{2}$, when $k_{p}-n+2 \leq h c \leq$ $k_{p}+n-1 ;$ and

- case IV: $P(h o p$ count $=h c)=1-A(h c-$ $\left.1, n, k_{p}, R\right) / \pi R^{2}$, when $h c=k_{p}+n$,

where $A\left(j, n, k_{p}, R\right)$ denotes the mean intersection area of two 


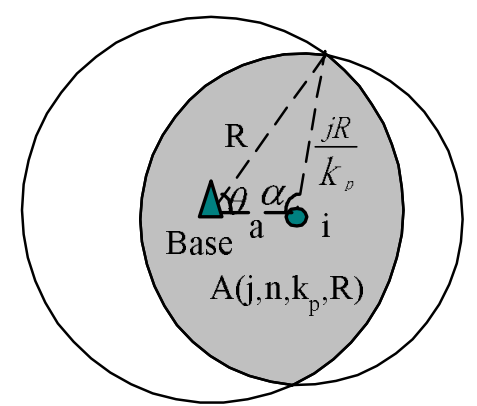

Fig. 8. Definition of $A\left(j, n, k_{p}, R\right)$.

circles with radiuses of $R$ and $j R / k_{p}$, i.e., the shaded area shown in Fig. 8. Since station $i$ is in the $n$th layer of the cell, we have $(n-1) \cdot R / k_{p}<a \leq n \cdot R / k_{p}$. Thus, we obtain $A\left(j, n, k_{p}, R\right)=\int_{(n-1) \cdot R / k_{p}}^{n R / k_{p}}\left[\alpha\left(j R / k_{p}\right)^{2}+\theta R^{2}-a R \sin \theta\right] d a$, where $\sin \theta=\left(j / k_{p}\right) \cdot \sin \alpha$, and $\cos \theta=\left(R^{2}+a^{2}-\right.$ $\left.\left(j R / k_{p}\right)^{2}\right) / 2 a R$ since $R^{2}-(R \cos \theta)^{2}=\left(j R / k_{p}\right)^{2}-(a-$ $R \cos \theta)^{2}$. Note that we take this example for $k_{p}=3$ and $n=2$ in Fig. 7 because it contains all kinds of cases.

Thus the average hop count from station $i$, located in the $n$th layer, to station $j$ in the same cell is $\sum_{h c=1}^{k_{p}+n} h c \cdot P($ hop count $=$ $h c)$. Note that the maximum hop count is $k_{p}+n$, i.e., $\mathrm{n}$ hops to the base and $k_{p}$ hops from the base to the station located near the boundary of the cell. Eventually, the mean hop count from station $i$, located randomly in the cell, to any destined station $j$ in the same cell is obtained as

$$
\begin{aligned}
& \text { avg_hc_ij }\left(k_{p}\right)= \\
& \quad \sum_{n=1}^{k_{p}} \frac{2 n-1}{k_{p}^{2}} \cdot \sum_{h c=1}^{k_{p}+n} h c \cdot P(\text { hop count }=h c) .
\end{aligned}
$$

It is simpler to derive the mean hop count from station $i$ to the base, $a v g_{-} h c_{-} i o\left(k_{p}\right)$, because the destination, i.e., the base, is in fixed position. Thus, avg $h c \_i o\left(k_{p}\right)$ can be derived as $\sum_{n=1}^{k_{p}}\left((2 n-1) / k_{p}^{2}\right) \cdot n$, which equals

$$
a v g_{-} h c_{-} i o\left(k_{p}\right)=\frac{\left(k_{p}+1\right)\left(4 k_{p}-1\right)}{6 k_{p}} \text {. }
$$

It is obvious that $a v g \_h c_{-} o i\left(k_{p}\right)$ equals $a v g \_h c_{-} i o\left(k_{p}\right)$ when the base sends a packet to any station $i$ in the cell.

System throughput analysis:

The throughput analysis process in $\mathrm{MCN}$ is similar to that in $\mathrm{SCN}$. Five major steps are required:

1. Derive $\lambda_{i}, G_{s}$, and $G_{b s}$ of $\mathrm{MCN}$;

2. Derive $t$ _idle to obtain the renewal interval;

3. Derive the probabilities of a successful transmission at station $i$ and at the base;

4. Derive the hop-by-hop throughput of station $i$ and the base; and

5. Derive the hop-by-hop throughput and end-to-end throughput of MCN.

Because stations help forward packets, the total traffic rate at

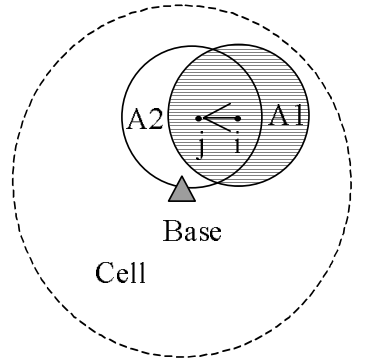

A1: capture area of station $i$

A2: hidden area of station $i$ for station $j$

Fig. 9. Capture area and hidden area of station $i$ in $\mathrm{MCN}$.

station $i$ is given as

$$
\begin{aligned}
\lambda_{i}= & \lambda_{i o} \cdot a v g_{-} h c_{-} i o\left(k_{p}\right)+\lambda_{o i} \cdot\left(a v g \_h c_{-} i o\left(k_{p}\right)-1\right) \\
& +\sum_{j \neq i, j=1}^{N} \lambda_{i j} \cdot a v g_{-} h c_{-} i j\left(k_{p}\right) .
\end{aligned}
$$

This is because each packet is, on average, forwarded either $a v g_{-} h c_{-} i j\left(k_{p}\right)$ times for intra-cell traffic, $a v g \_h c_{\_} i o\left(k_{p}\right)$ times for out-going inter-cell traffic, or $a v g h c \_i\left(k_{p}\right)-1$ times for incoming inter-cell traffic, in which the first transmission is done by the base. Notably, $\lambda_{i}$ is intuitively equivalent to the traffic rate handled, i.e., pumped or forwarded, by station $i$ because the traffic rate, to the cell, contributed by station $i$ should equal the traffic rate, contributed by others, station $i$ should take care of. Now we denote the traffic rate at all stations and the base by $G_{s}$ and $G_{b s}$, which equal $\sum_{i=1}^{N} \lambda_{i}$ and $\sum_{i=1}^{N} \lambda_{o i}$, respectively.

In MCN, t_idle is derived as $1 /\left(\left(G_{s}+G_{b s}\right) / k_{p}^{2}\right)$, which equals $k_{p}^{2} /\left(G_{s}+G_{b s}\right)$, since the area of a sub-cell is $1 / k_{p}^{2}$ of the area of a cell. The mean length of a renewal interval is thus defined as

$$
\begin{aligned}
& r n_{-} \text {cycle }= \\
& \quad l_{D I F S}+\frac{k_{p}^{2}}{G_{s}+G_{b s}}+l_{R T S}+r+l_{S I F S}+l_{C T S}+r \\
& +P s *\left(l_{S I F S}+l_{P K T}+r+l_{S I F S}+l_{A C K}+r\right) .
\end{aligned}
$$

For convenience, we again denote $n h$ and $h$ as $A 1 / \pi R^{2}$ and $A 2 / \pi R^{2}$, where $\mathrm{A} 1$ and $\mathrm{A} 2$ are the mean capture area and the mean hidden area, respectively, of station $i$ when station $i$ sends a packet to the next hop, say $j$, as shown in Fig. 9. The probability of a successful transmission at station $i$ can be derived similarly as done in SCN. However, to compute the success probability, three cases of the relative positions of station $i$ to the base have to be considered as shown in Fig. 10:

- case I:

$$
\begin{aligned}
P s_{i 1}= & \exp \left\{-\left[r \cdot\left(n_{-} h \cdot G_{s}+G_{b s}\right)\right.\right. \\
& \left.\left.+\left(2 r+l_{R T S}+l_{S I F S}\right) \cdot h \cdot G_{s}\right]\right\},
\end{aligned}
$$

when the distance between station $i$ and the base is smaller than $R / k_{p}$ so that the base is in the capture area of station $i$;

- case II:

$$
\begin{aligned}
P s_{i 2}= & \exp \left\{-\left[r \cdot n_{-} h \cdot G_{s}+\right.\right. \\
& \left.\left.\left(2 r+l_{R T S}+l_{S I F S}\right) \cdot\left(h \cdot G_{s}+P_{A} \cdot G_{b s}\right)\right]\right\},
\end{aligned}
$$




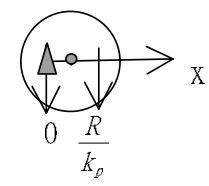

case I

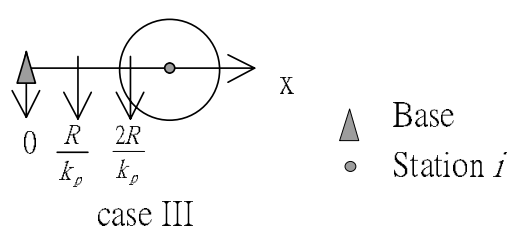

Fig. 10. Three cases to compute $P s_{i}$.

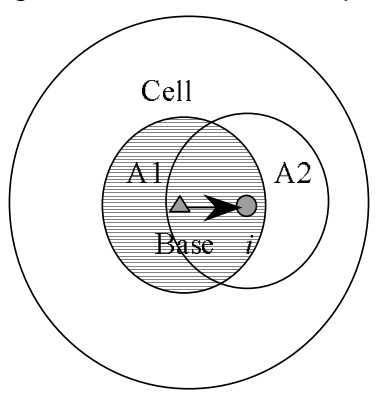

A 1: capture area of the base

A2: hidden area of the base for station $i$

Fig. 11. Capture area and hidden area of the base in MCN.

when the distance between station $i$ and the base is larger than $R / k_{p}$ but smaller than $2 R / k_{p}$ so that the base would be in the hidden area of station $i$, with probability $P_{A}$, when the receiver is positioned in the area $\mathrm{A}$ as shown in case II of Fig. 10; and

- case III:

$$
\begin{aligned}
P s_{i 3}= & \exp \left\{-\left[r \cdot n_{-} h \cdot G_{s}\right.\right. \\
& \left.\left.+\left(2 r+l_{R T S}+l_{S I F S}\right) \cdot h \cdot G_{s}\right]\right\},
\end{aligned}
$$

when the distance between station $i$ and the base is larger than $2 R / k_{p}$ so that the base does not affect the transmission of station $i$.

From the above discussion, we obtain that the mean probability of a successful transmission at station $i$ is

$$
P s_{i}=\frac{1}{k_{p}^{2}} \cdot P s_{i 1}+\frac{3}{k_{p}^{2}} \cdot P s_{i 2}+\frac{k_{p}^{2}-4}{k_{p}^{2}} \cdot P s_{i 3} .
$$

The derivation of the success probability at the base in MCN is similar to that in SCN, except that the hidden area, i.e., A2 in Fig. 11, within the cell should be considered. We thus obtain

$P s_{b s}=\exp \left\{-\left[r \cdot n_{-} h \cdot G_{s}+\left(2 r+l_{R T S}+l_{S I F S}\right) \cdot h \cdot G_{s}\right]\right\}$.

To get the probabilities that station $i$ and the base fire the first RTS packets within their sub-cells after a renewal point, we need to define the local traffic rate as the mean traffic rate within a sub-cell. Thus, the local traffic rates at station $i$ and at the base are $\lambda_{\text {local_ } i}=\left(G_{s}+G_{b s}\right) / k_{p}^{2}$ and $\lambda_{\text {local_bs }}=\left(G_{s} / k_{p}^{2}\right)+G_{b s}$, respectively. Notably, the term $G_{b s}$ in $\lambda_{\text {local_i }}$ is multiplied by
$1 / k_{p}^{2}$ because the probability that the base is in the sub-cell of station $i$ is $1 / k_{p}^{2}$. Hence, the probabilities for station $i$ and the base are $\lambda_{i} / \lambda_{\text {local_i } i}$ and $G_{b s} / \lambda_{\text {local_bs }}$, respectively. With these first transmission probabilities, the hop-by-hop throughput of station $i$ and the base are obtained as

$$
S_{i}=\frac{1 \cdot P s_{i}}{r n_{-} c y c l e} \cdot \frac{\lambda_{i}}{\lambda_{\text {local_i }}}
$$

and

$$
S_{b s}=\frac{1 \cdot P s_{b s}}{r n \_c y c l e} \cdot \frac{G_{b s}}{\lambda_{\text {local_bs }}}
$$

respectively.

Eventually, the hop-by-hop throughput of MCN is obtained as

$$
S_{h}=\sum_{i \in c e l l} S_{i}+S_{b s}
$$

The end-to-end throughput is derived as

$$
S_{e}=S_{h} \cdot F,
$$

where $F$ is given by

$$
F=\frac{A+B}{A \cdot a v g_{-} h c_{\_} i j\left(k_{p}\right)+(B+C) \cdot a v g \_h c_{\_} i o\left(k_{p}\right)},
$$

in which $A, B$ and $C$ are $\sum_{i=1}^{N} \sum_{j=1, j \neq i}^{N} \lambda_{i j}, \sum_{i=1}^{N} \lambda_{o i}$ and $\sum_{i=1}^{N} \lambda_{i o}$, respectively. Note that $A$ in denominator is multiplied by $a v g \_h c \_j\left(k_{p}\right)$. This is because in MCN each intra-cell packet is, on average, transmitted $a v g h c_{-} i j\left(k_{p}\right)$ times. For the same reason, $B$ and $C$ in denominator have to be multiplied by $a v g_{\_} h c_{-} i o\left(k_{p}\right)$. However, $C$ does not appear in numerator because the base is not the actual destinations of the inter-cell packets.

For the end-to-end throughput of MCN-b, the equations can be obtained by substituting $k_{p}$ by 1 and $R$ by $k_{b} \cdot R$. This is because, in MCN-b, the transmission range remains the same as that in SCN. However, the distance between bases is multiplied by $k_{b}$.

\section{NUMERICAL RESULTS}

The environmental difference between analysis and simulation is that, in simulation, the assumption, Route, is removed. Furthermore, in simulation, the position of each mobile station is randomly determined and the routing table is pre-computed by the all-pairs shortest path algorithm, Floyd-Warshall algorithm [16]. For simulation results, the SCN and MCN environments are simulated by PARSEC [17], a C based discrete-event simulation language developed at UCLA.

The values of $r, l_{P K T}, l_{A C K}, l_{R T S}, l_{C T S}$ are obtained by using the following parameter values: the data rate is $1.5 \mathrm{Mbps}$, propagation velocity of signals is $3 * 10^{8} \mathrm{~m} / \mathrm{s}$, and the lengths of a data, an acknowledgment, an RTS and an CTS packets are 1024, 14, 20, and 14 bytes, respectively. However, the value of propagation delay, $r$, is much smaller than the others and, therefore, is neglected in simulations. The other parameter values are as follows: the radius of a cell, $R$, is 150 meters, the 


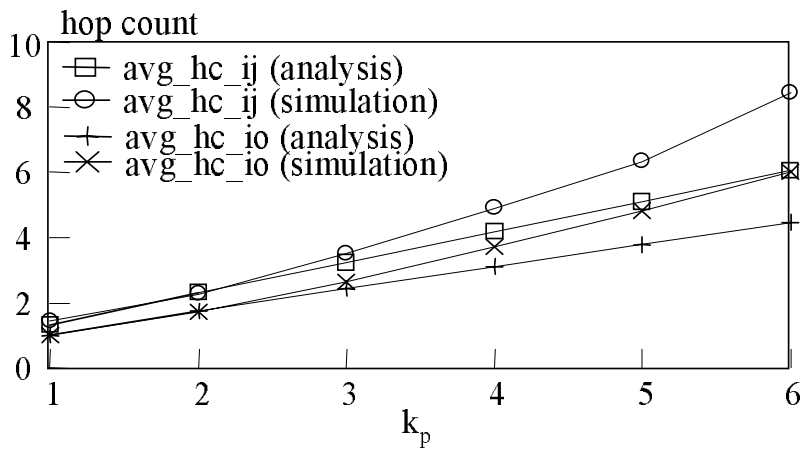

Fig. 12. Mean hop counts for intra-cell and inter-cell traffic in MCN.

number of mobile stations, $N$, is $250, l_{D I F S}=0.149 \mathrm{~ms}$, and $l_{S I F S}=0.042 \mathrm{~ms}$. The total traffic rate in a cell is 167 packets per second, which is about $1.5 \mathrm{Mbps}$ while considering DIFS, SIFS, RTS and CTS packets, and we normalize this traffic rate, $G$, to 1 , which is the default value.

Traffic locality, $\sum_{i=1}^{N} \sum_{j \neq i, j=1}^{N} \cdot \lambda_{i j} / \sum_{i=1}^{N} \lambda_{i}$, is defined to compare the end-to-end throughput of SCN and MCN, indicating the intra-cell traffic percentage of the traffic generated at station $i$. According to the values of the above parameters, we choose $\lambda_{i j}=0.0008955, \lambda_{i o}=0.223$ and $\lambda_{o i}=0.223$ so that locality $=0.5$, and the default value of locality is 0.5 .

\section{A. Mean hop count vs. mean number of channels in MCN}

We will first present the two important factors, mean hop count and mean number of channels, which significantly affect the throughput of MCN. Fig. 12 shows the results of mean hop count for intra-cell traffic, i.e., $a v g h c i j\left(k_{p}\right)$, and for inter-cell traffic, i.e., avg $h c_{-} i o\left(k_{p}\right)$, in $\mathrm{MCN}$. The curve for inter-cell traffic is lower than the curve for intra-cell traffic because the maximum hop count from station $i$ to the base is $R /\left(R / k_{p}\right)=k_{p}$ and the maximum hop count from station $i$ to station $j$ is $2 R /\left(R / k_{p}\right)=2 k_{p}$. For both cases, the hop count increases almost linearly as the transmission range decreases. When $k_{p}=1$, the value of $a v g \_h c_{\_} i o\left(k_{p}\right)$ equals 1 because each station $i$ can reach the base in a single hop. However, the value of $a v g \_h c_{-} i j\left(k_{p}\right)$ is in the interval between 1 and 2 because the hop count may be either 1 or 2 when station $i$ wants to send an intra-cell packet. The gap between simulation results and analysis results is owing to the Route assumption, i.e., station $i$ can always find the next hop, at a distance of $R / k_{p}$, in the straight-line direction towards the destination. The simulation model removes this assumption to reflect the real situation and examine its influence.

Fig. 13 shows that mean number of channels, $S_{h} \cdot$ rn_cycle, increases in square order, and mean hop count, i.e., (locality) . $a v g_{-} h c_{-} i j\left(k_{p}\right)+(1-l o c a l i t y) \cdot a v g \_h c_{-} i o\left(k_{p}\right)$, increases in linear order. Thus the positive side, as described in section I, dominates. This result can be explained by closely examining equations (2), (3), (5), and (6). The order of mean hop count, i.e., $\left[(\right.$ locality $) \cdot \sum_{n=1}^{k_{p}}\left((2 n-1) / k_{p}^{2}\right) \cdot \sum_{h c=1}^{k_{p}+n} h c \cdot P($ hop count $=h c)]+\left[(1-\right.$ locality $\left.) \cdot\left(\left(k_{p}+1\right)\left(4 k_{p}-1\right) / 6 k_{p}\right)\right]$ from equations (2) and (3), is close to $k_{p}$. However, the order of mean number of channels, i.e., (5) $\cdot(6)=k_{p}^{2} \cdot\left[P s_{i} \cdot G_{s} /\left(G_{s}+G_{b s}\right)+\right.$

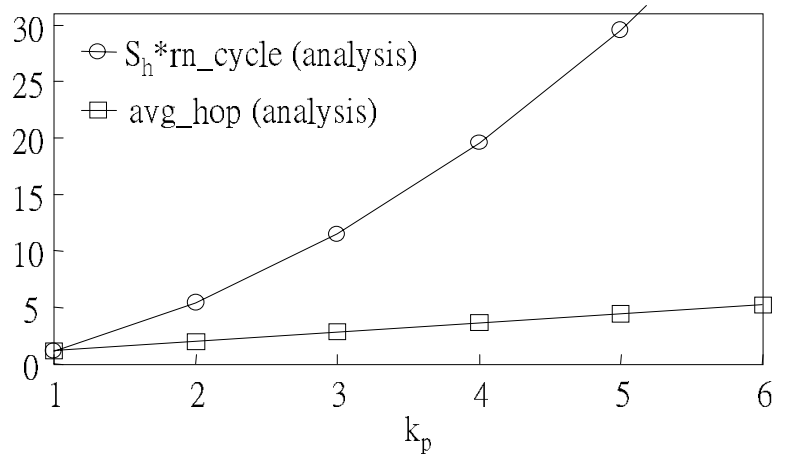

Fig. 13. Mean number of channels vs. mean hop count. number of packets / renewal cycle

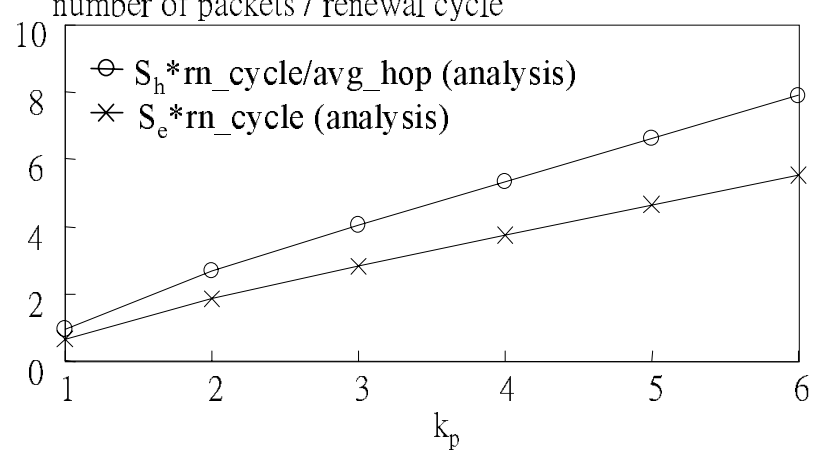

Fig. 14. End-to-end throughput vs. ratio of mean number of channels to mean hop count.

$\left.P s_{b s} \cdot G_{b s} /\left(G_{s}+k_{p}^{2} G_{b s}\right)\right]$ from equations (5) and (6), is close to $k_{p}^{2}$ because $G_{s}$ is usually much larger than $G_{b s}$.

Fig. 14 shows the number of simultaneously received packets at destinations per renewal cycle, i.e., $S_{e} \cdot r n_{-} c y c l e$, and the mean number of channels divided by mean hop count. Because of the lack of the term, $C$, in numerator in equation (7), there is some over-estimation in the latter curve; otherwise, the two curves will coincide with each other. This reveals that the increasing order of end-to-end throughput is mainly affected by mean hop count.

\section{B. $S C N$ vs. $M C N$}

Of particular interest is the difference of the throughput between SCN and MCN. As shown in Fig. 15, even when the transmission range of the base and mobile stations in MCN are the same as those in SCN, i.e., $k_{p}=1$ in MCN, the throughput in MCN performs slightly better than that in SCN. This is mainly because of the key feature in MCN that mobile stations can communicate with each other.

When all traffic are inter-cell traffic, i.e., locality $=0$, the throughput in SCN and MCN are the same. In this case, all packets arrive in the base in one hop. However, when all traffic are intra-cell traffic, i.e., locality $=1, \mathrm{MCN}$ has better throughput performance. This is because in SCN, each packet is transmitted exactly twice, one for upstream and the other for downstream. However, in $\mathrm{MCN}$, because of the key feature, each packet is transmitted either once or twice. Both the curves of "MCN minus SCN" show that the gain of the feature in MCN depends on locality. 
end-to-end throughput: $\mathrm{S}_{\mathrm{e}}$ (Mbps)

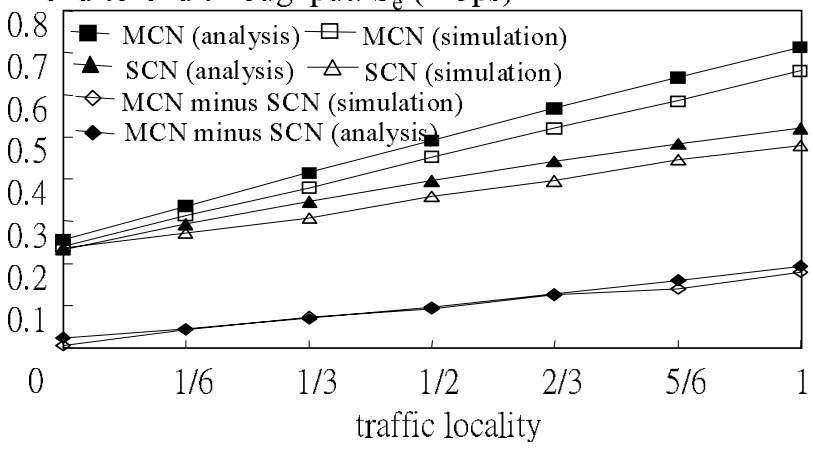

Fig. 15. Throughput comparison between SCN and MCN for $k_{p}=1$. end-to-end throughput: Se (Mbps)

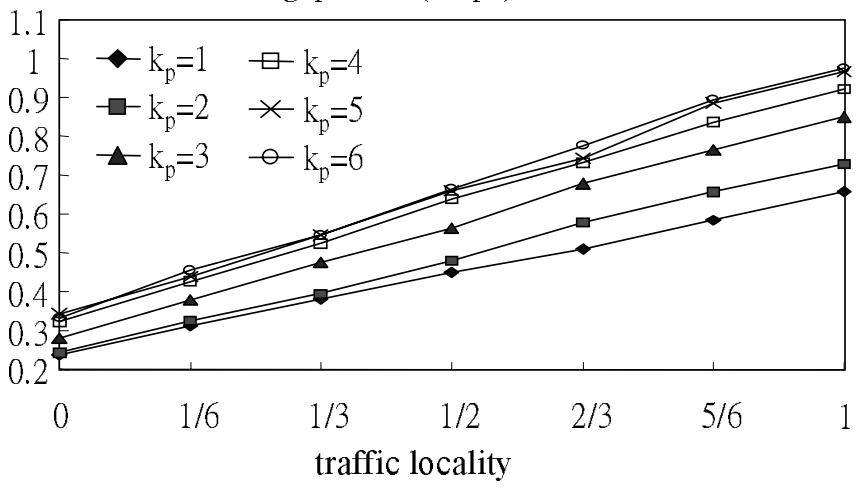

Fig. 16. Impact of traffic locality on throughput for various $k_{p}$ in MCN.

From the derived formulas of $G_{b s}$ and end-to-end throughput of SCN and for the lack of the term, $C$, in numerator in equation (7), we can see that higher locality increases throughput of SCN and MCN, respectively. However, the end-to-end throughput of simulation results are lower than those of analysis results is owing to the higher value of mean hop count of simulation results, as shown in Fig. 15.

Fig. 16 shows the simulated throughput in MCN for different value of $k_{p}$ under various locality. When all traffic are inter-cell traffic, i.e., locality $=0$, the throughput increase for larger $k_{p}$ is slight. This is because, as $k_{p}$ increases, the increase of the traffic generating rates, i.e., $\lambda_{i}$, at mobile stations residing within the sub-cell of the base will pump more traffic, and, therefore, increase the throughput. However, because the base becomes the bottleneck, the throughput increases slightly as $k_{p}$ increases. However, as discussed above, the throughput increases apparently when locality increases. Thus, Fig. 16 shows that MCN is more suitable for the LAN environment, where the intra-LAN traffic dominates, than for the WAN environment, where the transit, i.e., inter-LAN, traffic dominates.

\section{C. $M C N-p$ vs. $M C N-b$}

In this subsection, the throughput performance of MCN-p and MCN-b are compared. From Fig. 1 we can see that the area of a cell in MCN-b is larger than that in MCN-p. When the numbers of mobile stations in a cell of the two architectures are the same, the throughput will be slightly different because of different propagation delay. The results are shown by the two

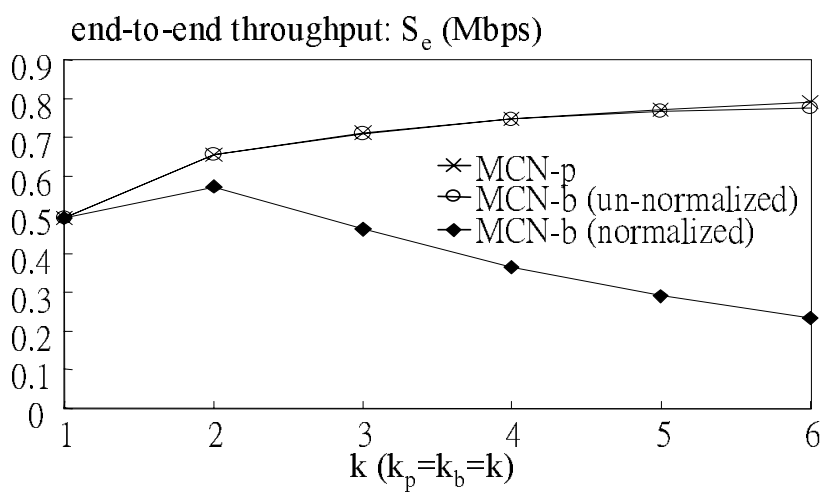

Fig. 17. The difference between MCN-p and MCN-b.

coincide curves, "MCN-p" and "MCN-b (un-normalized)," in Fig. 17.

However, when the densities of mobile stations of the two architectures are the same, the throughput in MCN-b descends quickly as shown by the "MCN-b (normalized)" curve in Fig. 17. This is because that the transmission range in MCN-b is $k_{p}$ times of that in MCN-p, i.e., the number of mobile stations in a sub-cell in MCN-b is $k_{p}^{2}$ times of that in MCN-p. Hence, the offered traffic within a sub-cell in MCN-b is also $k_{p}^{2}$ times of that in MCN-p. This causes the probability of a successful transmission decreases rapidly in MCN-b. We conclude that given the densities of bases, i.e., $R$, mobile stations, i.e., $N$, and traffic, i.e., $\lambda$, our formulas help determine the transmission range for the desired throughput level.

\section{CONCLUSION}

This work presents a novel architecture, Multihop Cellular Network (MCN), and derives the throughput of MCN and its counterpart, Single-hop Cellular Network (SCN), based on the RTS/CTS access method. The throughput is analyzed by using the technique of renewal process, in which the renewal point is defined as the time point when all stations in a sub-cell simultaneously sense that the channel is idle. Furthermore, mean hop count is analyzed because it significantly influences the throughput of MCN, as confirmed by the numerical results.

The analysis and simulation results of the throughput of SCN and MCN lead to four important observations. First, the throughput of MCN is superior to that of the corresponding SCN. Second, the throughput of MCN increases as the transmission range decreases. We explain these two observations by illustrating the different increasing orders, $k_{p}^{2}$ and $k_{p}$, respectively, of mean number of channels, i.e., simultaneous transmissions in a cell, and mean hop count, as the transmission range decreases by $k_{p}$ times. Third, MCN is more suitable for the LAN environment, where the intra-LAN traffic dominates, than for the WAN environment, where the transit, i.e., inter-LAN, traffic dominates. Fourth, given the densities of bases, i.e., $R$, stations, i.e., $N$, and traffic, i.e., $\lambda$, our formulas help determine the transmission range for the desired throughput level. When the transmission range and distance between bases in MCN-b are both $k_{p}$ times of those in MCN-p, the number of stations in a sub-cell becomes $k_{p}^{2}$ times of that in MCN-p, and, therefore, 
the throughput performance descends quickly.

Although MCN shows a higher throughput than SCN, some related issues must be further studied. The first thing is how to obtain an appropriate operational value of $k_{p}$ while considering both throughput performance which favors large $k_{p}$ and path vulnerability which favors small $k_{p}$. Furthermore, the mobility of stations cannot be neglected in routing protocol. Thus, future research should develop an efficient routing algorithm and more closely examine the issues of handoff and mobility management in the MCN environment.

\section{REFERENCES}

[1] K. C. Budka, "Cellular digital packet data-advanced mobile phone standard network bandwidth contention," in Proc. the 34th IEEE Conf. Decision and Control, pp. 1941-1946, New Orleans, Dec. 1995.

[2] J. Cai and D. J. Goodman, "General packet radio service in GSM," IEEE Commun. Mag., vol. 35, no. 10, pp. 122-131, Oct. 1997.

[3] T. Wilkinson, T. G. C. Phipps, and S. K. Barton, "A report on HIPERLAN standardization," Int. J. Wireless Inform. Networks, vol. 2, no. 2, pp. 99$120,1995$.

[4] IEEE Standards Board, "Part 11: Wireless LAN medium access control (MAC) and physical layer (PHY) specifications," The Institute of Electrical and Electronics Engineers, Inc., IEEE Std 802.11-1997.

[5] J. Jubin and J. D. Tornow, "The DARPA packet radio network protocols," IEEE Proc., vol. 75, no. 1, Jan. 1987.

[6] B. M. Leiner et al., "Issues in packet radio network design," IEEE Proc., vol. 75 , no. 1 , Jan. 1987.

[7] A. Alwan et al., "Adapting to a highly variable and unpredictable environment: adaptive mobile multimedia networks," IEEE Personal Commun. pp. 34-51, Apr. 1996.

[8] Y. D. Lin et al.,"Multihop wireless IEEE 802.11 LANs: a prototype implementation," in Proc. IEEE ICC'99, Vancouver, Canada, June 1999.

[9] Available at http://www.metricom.com/.

[10] I. F. Akyildiz, W. Yen, and B. Yener, "A new hierarchical routing protocol for dynamic multihop wireless networks," in Proc. IEEE INFOCOM'97, 1997.

[11] Vodafone and Salbu, "ODMA-opportunity driven multiple access," SMG2 UMTS Workshop, Sophia Antipolis, 1996.

[12] F. A. Tobagi and L. Kleinrock, "Packet switching in radio channels, Part II: The hidden-terminal problem in carrier sense multiple access and the bus-tone solution," IEEE Trans. Commun., vol. 23, pp. 1417-1433, 1975.

[13] Z. J. Haas, "On the performance of a medium access control scheme for the reconfigurable wireless networks," in Proc. MILCOM'97, pp. 1558$1564,1997$.
[14] H. S. Chhaya and S. Gupta, "Performance modeling of asynchronous data transfer methods of IEEE 802.11 MAC protocol," Wireless Networks, vol. 3, pp. 217-234, 1997.

[15] T.-C. Hou and V. O. K. Li, "Transmission Range Control in Multihop Packet Radio Networks," IEEE Trans. Commun., vol. 34, pp. 38-44, 1986.

[16] T. H. Cormen, C. E. Leiserson, and R. L. Rivest, "Introduction to Algorithms," The MIT Press, pp. 558-565, 1992.

[17] R. A. Meyer, "PARSEC user manual," Available at http://pcl.cs.ucla.edu/, Aug. 1998.

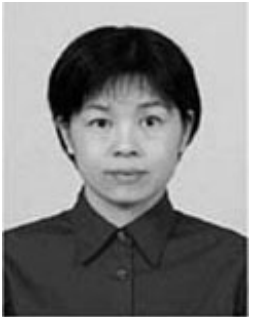

Yu-Ching Hsu was born in Tei-Tou, Taiwan in 1973. She received her B.A. and M.S. in Computer and Information Science from National Chiao Tung University in 1995 and 1997, respectively. She is currently a $\mathrm{Ph} . \mathrm{D}$. candidate in the same department. Her research interests include protocol design, analysis, and wireless communications. She can be reached at ychsu@cis.nctu.edu.tw.

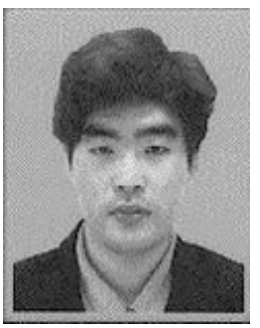

Ying-Dar Lin was born in Hsi-Lo, Taiwan in 1965 He received the Bachelor's degree in Computer Science and Information Engineering from National Taiwan University in 1988, and the M.S. and Ph.D. degrees in Computer Science from the University of California, Los Angeles in 1990 and 1993, respectively. At UCLA Computer Science Department, he worked as a Research Assistant from 1989 to 1993 and worked as a Teaching Assistant from 1991 to 1992 . In the summers of 1987 and 1991, he was a technical staff member in IBM Taiwan and Bell Communications Research, respectively. He joined the faculty of the Department of Computer and Information Science at National Chiao Tung University in August 1993 and is Professor since 1999. His research interests include design, analysis, and implementation of network protocols and algorithms, wire-speed switching and routing, quality of services, and intranet servers. He has been a consultant for several high-tech companies and authored two books including a textbook on Computer Network Experiments. He is a member of ACM and IEEE. He can be reached at ydlin@cis.nctu.edu.tw and http://speed.cis.nctu.edu.tw/_ydlin/. 